\title{
Cellular localization and biochemical characterization of a novel calcium- dependent protein kinase from tobacco
}

\author{
Yun WANG", Mei ZHANG", Ke KE, Ying Tang LU* \\ Key Lab of MOE for Plant Developmental Biology, College of Life Sciences, Wuhan University, Wuhan 430072, China
}

\begin{abstract}
By screening tobacco cDNA library with $M C K 1$ as a probe, we isolated a cDNA clone $N t C P K 5$ (accession number AY 971376), which encodes a typical calcium-dependent protein kinase. Sequence analyses indicated that NtCPK5 is related to both CPKs and CRKs superfamilies and has all of the three conserved domains of CPKs. The biochemical activity of NtCPK 5 was calcium-dependent. NtCPK5 had $V_{\max }$ and $K_{m}$ of $526 \mathrm{nmol} / \mathrm{min} / \mathrm{mg}$ and $210 \mu \mathrm{g} / \mathrm{ml}$ respectively with calf thymus histone (fraction III, abbreviated to histone IIIs) as substrate. For substrate syntide-2, NtCPK 5 showed a higher $V_{\max }$ of $2008 \mathrm{nmol} / \mathrm{min} / \mathrm{mg}$ and a lower $K_{m}$ of $30 \mu \mathrm{M}$. The $K_{0,5}$ of calcium activation was $0.04 \mu \mathrm{M}$ or $0.06 \mu \mathrm{M}$ for histone IIIs or syntide- 2 respectively. The putative myristoylation and palmitoylation consensus sequence of NtCPK5 suggests that it could be a membrane-anchoring protein. Indeed, our transient expression experiments with wild type and mutant forms of NtCPK5/GFP fusion proteins showed that NtCPK 5 was localized to the plasma membrane of onion epidermal cells and that the localization required the N-terminal acylation sites of NtCPK5/GFP. Taking together, our data have demonstrated the biochemical characteristics of a novel protein NtCPK 5 and its subcellular localization as a membrane-anchoring protein.
\end{abstract}

Keywords: calcium-dependent protein kinase, phosphorylation, GFP, subcellular localization.

\section{INTRODUCTION}

Calcium $\left(\mathrm{Ca}^{2+}\right)$ as a ubiquitous signal molecule plays vital roles in plant response to various stimuli, including light, environmental stresses, pathogen attack, and hormones by fluctuation of cytosolic $\mathrm{Ca}^{2+}$ concentration in all living cells [1]. In plants, different calcium signal was transduced through several calcium-binding proteins such as calmodulin $(\mathrm{CaM})$, calcium-dependent protein kinase (CDPK or CPK), calcineurin B-like protein (CBL) and chimeric $\mathrm{Ca}^{2+} / \mathrm{CaM}$-dependent protein kinase (CCaMK) into downstream responses, including altered protein phos-

\footnotetext{
TThese authors made equal contribution to the paper.

*Correspondence: Ying Tang LU

Tel: +86-27-68752619; Fax: +86-27-68756380;

E-mail: yingtlu@whu.edu.cn

Abbreviations: CPK or CDPK ( $\mathrm{Ca}^{2+}$-dependent protein kinase); CRK (CPK-related kinase); $\mathrm{CBK}$ (calmodulin-binding protein kinase); CaMK $\left(\mathrm{Ca}^{2+} / \mathrm{CaM}\right.$-dependent protein kinase); $\mathrm{CCaMK}$ (chimeric $\mathrm{Ca}^{2+} / \mathrm{CaM}$ dependent protein kinase); MCK (maize homologue of mammalian CaMK); CBL (calcineurin B-like protein); GFP (green fluorescent protein); histone IIIs (calf thymus histones fraction III); Ni-NTA $\left(\mathrm{Ni}^{2+}\right.$ nitrilotriacetate).
}

phorylation and gene expression patterns [2-4]. CPKs are a group of well-characterized protein kinases and have been found in many plants including Arabidopsis, soybean, rice, tobacco and potato [5]. CPKs contain three conserved domains: kinase catalytic domain, junction domain and calmodulin-like domain. Junction domain can serve as a pseudosubstrate and block the catalytic pocket of kinase domain in the absence of calcium. Upon the calcium binding to EF-hands, junction domain (the autoinhibitory region) is released from the kinase domain to activate CPKs [6].

Except of the three highly conserved domains, CPKs also have an $\mathrm{N}$-terminal variable region, which often contains N-terminal acylation (including myristoylation and palmitoylation) sites required for the subcellular localization of certain CPKs [7-10]. Myristoylation often occurs at Gly residue at position 2 and may or may not sufficient for membrane anchoring. Palmitoylation occurred at Cys residue at position 4 as a supplement can promote and stabilize the targeting of CPKs to plasma membrane [7-9]. In Arabidopsis, there are $34 \mathrm{CPK}$ isoforms, among them, only five AtCPKs lack both Gly2 and Cys4. Different N-terminal acylation from variable $\mathrm{N}$-terminal sequences produces isoform-specific subcellular localization of AtCPKs, sug- 
gesting that this family of protein kinases may be involved in multiple signaling pathways due to differential distribution in cells [10].

In this paper, we reported the cloning of $N t C P K 5$, a new $C P K$ isoform from tobacco plants and the biochemical characterization of $\mathrm{NtCPK} 5$ activity. NtCPK 5 shares high sequence homology to NtCPK4. However their variable N-terminal regions are diverse. Our experiments showed that these two tobacco NtCPK isoforms have different subcellular distribution and that the two amino acids Gly2 and Cys 4 of NtCPK 5 are crucial for its membrane localization.

\section{MATERIALS AND METHODS \\ Isolation of the cDNA encoding NtCPK5}

A tobacco cDNA library was constructed from mRNAs isolated from leaves of Nicotiana tabaccum cv. W38 using ZAPcDNA Synthesis Kit, following the manufacturer's instructions (Invitrogene). The library was then screened using maize $M C K 1$ cDNA [11] as a probe, and a positive plasmid containing $N t C P K 5$, named $\mathrm{p} N t C P K 5$ was isolated and sequenced.

\section{Construction of plasmids NtCPK5 and truncated NtCPK5 $P 1$ and $P 2$}

To identify the kinase properties of $\mathrm{NtCPK} 5$, several constructs were made in plasmid pFastHTb following the protocol described previously $[12,13]$. Using $\mathrm{pNtCPK5}$ as template, the cDNAs for the full open reading frame (NtCPK5) and two truncated forms P1 (residues 1-372) and P2 (residues 1-408) of NtCPK5 were amplified and purified. $\mathrm{P} 1$ contains the $\mathrm{N}$-terminal 372 amino acids without the C-terminal calmodulin like domain and junction domain of NtCPK5 while $\mathrm{P} 2$ contains the N-terminal 408 amino acids without the $\mathrm{C}$ terminal calmodulin like domain of NtCPK5 only. The PCR products were digested with HindIII and cloned into the HindIII site of plasmid pFastBacHTb. The orientation of the insertion was checked by restriction digestion.

The recombinant plasmids were sequenced and transformed into DH10Bac competent cells containing the bacmids with a mini-att Tn7 target site and helper plasmid. The mini Tn7 element on the pFastBacHTb donor plasmid can transpose to the mini-att $\operatorname{Tn} 7$ element on the bacmid in the presence of transposition proteins provided by the helper plasmid. Clones containing recombinant bacmid were identified based on the disruption of the lac $Z$ gene. The sf- 9 cells were maintained as mono-layer at $27^{\circ} \mathrm{C}$ in $10 \%$ fetal bovine serum supplement with Grace's medium and transfected with the recombinant bacmid with CELLFECTIN reagent according to manufacturer's instructions (Invitrogen). Recombinant virus were harvested after $72 \mathrm{~h}$ and identified by PCR, then was used in following assay or saved at $-80^{\circ} \mathrm{C}$.

\section{Purification of recombinant proteins}

The NtCPK5 protein expression in Insect sf-9 cells and protein purification were performed as described previously [14, 15]. In briefly, sf- 9 cells were harvested at room temperature after infected by the recombinant virus for $72 \mathrm{~h}$. The cells were washed once with Grace's medium, resuspended in $5 \mathrm{ml}$ of lysis buffer [50 mM Tris$\mathrm{HCl}(\mathrm{pH} 7.5), 10 \%$ (v/v) glycerol, $1 \%$ Nonidet P40, $0.2 \mathrm{mM}$ PMSF], and then homogenized by sonication for $30 \mathrm{~s}$, followed by centrifugation at $12000 \mathrm{~g}$ for $10 \mathrm{~min}$. The supernatant was applied to a NiNTA $\left(\mathrm{Ni}^{2+}\right.$-nitrilotriacetate) resin column pre-equilibrated with buffer A [50 mM potassium phosphate ( $\mathrm{pH} 6.0), 300 \mathrm{mM} \mathrm{KCl}, 10 \%$ glycerol]. After extensively washing with buffer A and buffer A containing $25 \mathrm{mM}$ imidazole, recombinant proteins were subsequently eluted with buffer A containing $100 \mathrm{mM}$ imidazole. The eluted recombinant proteins were dialyzed against $25 \mathrm{mM}$ Tris-HCl, $\mathrm{pH} 7.5$ for approximately $6 \mathrm{~h}$, and then used for SDS-PAGE and enzymatic analyses. Protein concentration was determined by the method of Bradford using BSA as a standard. All procedures were performed at $4^{\circ} \mathrm{C}$, unless stated otherwise.

\section{Kinase assays}

Autophosphorylation and substrate phosphorylation assays were carried out in kinase buffer containing $25 \mathrm{mM}$ Tris- $\mathrm{HCl}, \mathrm{pH}$ 7.5, 0.5 $\mathrm{mM}$ dithiothreitol, $10 \mathrm{mM} \mathrm{MgCl}{ }_{2}, 50 \mu \mathrm{M}$ ATP, $10 \mu \mathrm{Ci}\left[\gamma_{-}{ }^{32} \mathrm{P}\right]$ ATP $(5000 \mathrm{Ci} / \mathrm{mM}), 0.1 \mathrm{mM} \mathrm{CaCl}_{2}$ or $2 \mathrm{mM} \mathrm{EGTA}$ at $30^{\circ} \mathrm{C}$ for $30 \mathrm{~min}$, with histone IIIs as substrate $(1 \mathrm{mg} / \mathrm{ml})$. The reactions were initiated by addition of $100 \mathrm{ng} \mathrm{NtCPK5,P} 1$ or P2, terminated by adding $1 / 5$ volume $5 \times$ Laemmli Sample Buffer, and analyzed by $10 \%$ SDSPAGE. After staining with $0.1 \%$ Coomassie Brilliant Blue, the gels were vacuum-dried and exposed to $\mathrm{x}$-ray film at $-80^{\circ} \mathrm{C}$ with a screen.

The kinase activities were measured under different $\mathrm{pH}(\mathrm{pH}$ 6-9.5) and $\mathrm{Mg}^{2+}$ concentration $(0-25 \mathrm{mM})$, or different reaction times $(0-60$ $\mathrm{min})$. Aliquots $(50 \mu \mathrm{l})$ were terminated by adding a $1 / 5$ volume of $5 \times$ Laemmli Sample Buffer, and analyzed by SDS-PAGE. After staining with $0.1 \%$ Coomassie Brilliant Blue, the substrate bands (histone IIIs) were collected and the ${ }^{32} \mathrm{P}$ incorporation was determined by liquid scintillation counting (Beckman LS 6500). Enzyme assays were also performed in the presence of different concentrations of $\mathrm{Ca}^{2+}$ (10 nM-60 $\mu \mathrm{M}$ with histone IIIs or syntide-2 as substrates), histone IIIs $\left(0.01-1 \mathrm{mg} / \mathrm{ml}\right.$ with $\left.0.1 \mathrm{mM} \mathrm{CaCl}_{2}\right)$ and syntide-2 $(1-150 \mu \mathrm{M}$ with $0.1 \mathrm{mM} \mathrm{CaCl}$ ). For $\mathrm{Ca}^{2+}$-dependent curves, free $\mathrm{Ca}^{2+}$ levels were set using $\mathrm{Ca}^{2+} /$ EGTA buffer as described by Bers [16]. When using syntide- 2 as substrate, the reaction mixture was pipetted onto squares of $2 \times 2 \mathrm{~cm}$ phosphocellulose paper (type P81, Whatman) and then the paper was immediately immersed into $75 \mathrm{mM}$ phosphoric acid, followed by five times wash 5 min each in $75 \mathrm{mM}$ phosphoric acid and one time wash in acetone, and then dried and counted by liquid scintillation.

\section{Construction of GFP fusion proteins}

DNA fragments containing ORFs for both NtCPK5 and NtCPK4 were obtained by PCR with forward primers (GGA TCC ATG GGC AGC TGT TTT TCT AGC TCC and GGA TCC ATG GGT AAT AAC TGT TTT TCT AGC) and reverse primers (GGA TCC CAA AGC TAC ATT TCT CCG TGA ATC and GGA TCC ACT TTT CCG AGA GCC TCT AAC AG). The full length cDNAs of NtCPK5 with mutated myristoylation site (Gly2Ala) or mutated palmitoylation site (Cys4Ala) or mutated both myristoylation and palmitoylation sites (Gly2Ala/Cys4Ala) were PCR generated by in vitro mutagenesis with site-mutated forward primers (GGA TCC ATG GCC AGC TGT TTT TCT AGC TCC, or GGA TCC ATG GGC AGC GCC TTT TCT AGC TCC, or GGA TCC ATG GCC AGC GCC TTT TCT AGC TCC) respectively together with reverse primer (GGA TCC CAA AGC TAC ATT TCT CCG TGA ATC). All of these PCR products were cloned in pGM T-vector, digested and further cloned into the $\mathrm{pUC} / \mathrm{GFP}$ made by ligating $35 \mathrm{~S}$ promoter 
from pBIm [17] and GFP from pBI101-GFP [17] into pUC18 in frame to produce C-terminal GFP tagged fusion protein. After sequence confirmation, these vectors were bombarded into the epidermal cells of onion.

\section{Transient expression of NtCPK4/GFP, NtCPK5/GFP and three mutation forms of NtCPK5/GFP}

The experiments were performed as previously described by Scott [18]. The inner epidermal peels of onion (Allium cepa) buld $(2 \times 2 \mathrm{~cm})$ were riped and placed on agar plates containing $1 \times$ Murashige andSkoog (MS) salts, $30 \mathrm{~g} / \mathrm{L}$ sucrose and $3 \%$ agar, $\mathrm{pH}$ 5.7. Peels were bombarded within $1 \mathrm{~h}$ after transferred to agar plates. The five constructs (including NtCPK4/GFP, NtCPK5/GFP, Gly2Ala NtCPK5/ GFP, Cys4Ala NtCPK5/GFP, and Gly2Ala/Cys4Ala NtCPK5/GFP) were delivered into onion epidermal cells with a biolistic PDS-1000/ HeTM (BioRad) particle gun with 1100 psi rupture discs under a vacuum of 28 in $\mathrm{Hg}$ [18]. Three bombardments were performed for each construction. Onion epidermal cells were allowed to recover for about $20 \mathrm{~h}$ at $22^{\circ} \mathrm{C}$ in continual light before they were analyzed by confocal laser scanning microscopy at $488 \mathrm{~nm}$ (Leica, TCS-SP).

\section{RESULTS}

\section{Molecular cloning of NtCPK5}

To clone a maize $M C K 1$ ortholog in tobacco, a tobacco cDNA library was screened and a cDNA clone, named $N t C P K 5$, was isolated. The NtCPK5 cDNA (accession number AY971376) is 2368 bp long with an open reading frame (ORF) of $1704 \mathrm{bp}$ encoding 567 amino acids with a molecular weight of $64 \mathrm{kDa}$ (Fig. 1). NtCPK5 belongs to CPKs superfamily [5, 6], comprising an N-terminal variable region, a kinase domain, a junction domain, and a $\mathrm{C}$ terminal calmodulin-like domain with four EF-hand motifs implicated in $\mathrm{Ca}^{2+}$ binding. The kinase domain (266 amino acids) of NtCPK 5 contains all of the 11 conserved subdomains of Ser/Thr protein kinases including a putative ATP-binding site in the N-terminal region and a Ser/ $\mathrm{Thr}$ protein kinase active site in the center of the kinase domain. By searching ScanProsite in PlantsP database [19], seven putative $\mathrm{N}$-myristoylation sites are found in $\mathrm{NtCPK} 5$ as shown in Fig. 1 with underlines. The N-terminal putative myristoylation site (MGxxxSxx, PlantsP database) is conserved within plant CPKs superfamily [7-10], which may be required for the subcellular localization of NtCPK5.

The amino acid sequence of NtCPK 5 was aligned with those of other plant CPKs and CRKs by using DNAstar alignment program (Fig. 2A and $2 \mathrm{~B}$ ). The results show that $\mathrm{NtCPK} 5$ is highly identical to other CPKs. It shares $87.1 \%$ and $89.4 \%$ amino acid sequence identity to that of $\mathrm{NtCPK} 4$ (lab data) and of StCPK1 [20] respectively. Except of NtCPK4 and StCPK1, other CPKs including NtCDPK1 (accession number AF072908, 42.6\% identity) [21] and NtCDPK2 (accession number AJ344154, 40.6\% identity) [22] in tobacco, LeCPK1 in tomato (accession number AJ308296, 42.3\% identity) [8], and CpCPK1 in

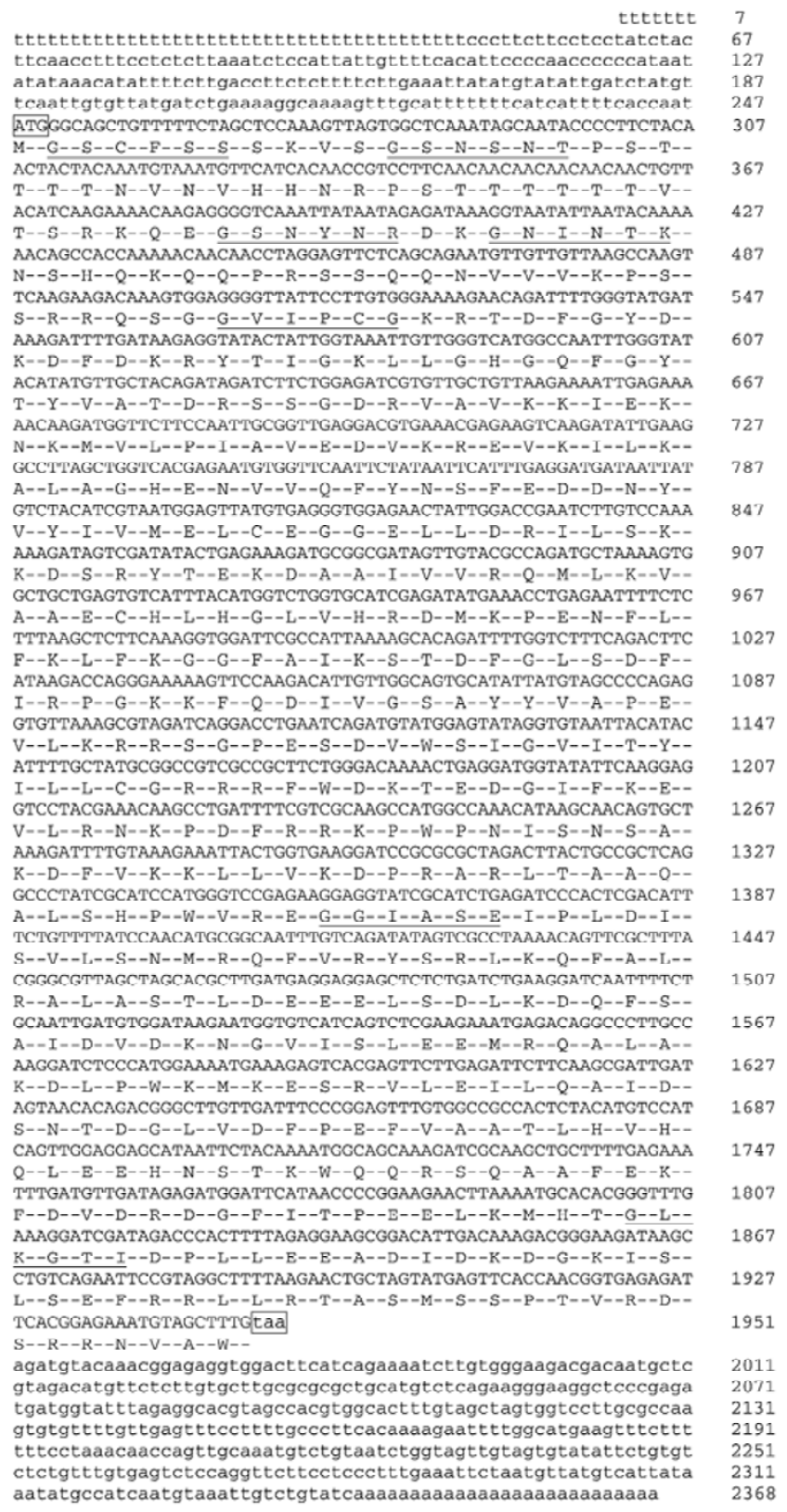

Fig. 1 Nucleotide and deduced amino acid sequences of NtCPK5. The nucleotides are numbered on the right. The start codon (ATG) and the stop codon (taa) in frame are boxed respectively. The underlined sequences are putative myristoylation consensus predicted by ScanProsite in PlantsP.

zucchini (accession number U90262, 41.5\% identity) [23] also share sequence homology to NtCPK5. Phylogenic tree analyses indicate that $\mathrm{NtCPK} 5$ subfamily including $\mathrm{NtCPK} 4$ and StCPK1 is more related to CRK than to CPK. The sequence identity between NtCPK 5 and CRK proteins such as maize MCK1 (accession number 1839597) [11] and 
ZmCRK (accession number D84507), carrot DcCRK (accession number X83869) [24], and Arabidopsis AtCRK1 (accession number AF435448) [13] are 43.3\%, $43.3 \%, 44.3 \%$, and $44.3 \%$ respectively. NtCPK 5 may be thus considered as an evolutionary link between CPKs and CRKs (Fig. 2B).

A putative myristoylation site (MGxxxSxx, PlantsP database) at the beginning of the NCPK5 coding region, which is also present in other plant CPKs [7-10], was presumed involving in the subcellular localization of NtCPK5.

\section{Analysis of the kinase activity of NtCPK5 protein}

To characterize the biochemical activity of NtCPK 5 as a calcium-dependent protein kinase, the full length as well as two truncated proteins of NtCPK 5 was tagged with HT and the recombinant proteins were expressed in sf-9 cells (Fig. 3A). The proteins were purified, checked on an SDSPAGE (Fig. 3B), and used for enzymatic analyses.

The full-length NtCPK 5 has autophosphorylation activity. The phosphorylation occurred in the presence of $0.1 \mathrm{mM} \mathrm{Ca}^{2+}$ (Fig. 3C). Addition of EGTA greatly reduced the autophosphorylation of NtCPK5. NtCPK 5 is an active kinase towards histone IIIs in the presence of $\mathrm{Ca}^{2+}$. Similar to its autophosphorylation activity, the presence of EGTA decreased the kinase activity of NtCPK5, similar to other known CPKs [21].

To examine the possible role of the putative junction domain and calmodulin-like domain in regulation of the kinase activity of $\mathrm{NtCPK} 5$, two truncated forms of NtCPK5, P1 (residues 1-372) and P2 (residues 1-408) were used in the kinase assay. P1 was expressed and purified as the full-length NtCPK5 protein (Fig. 3B). It con-

A

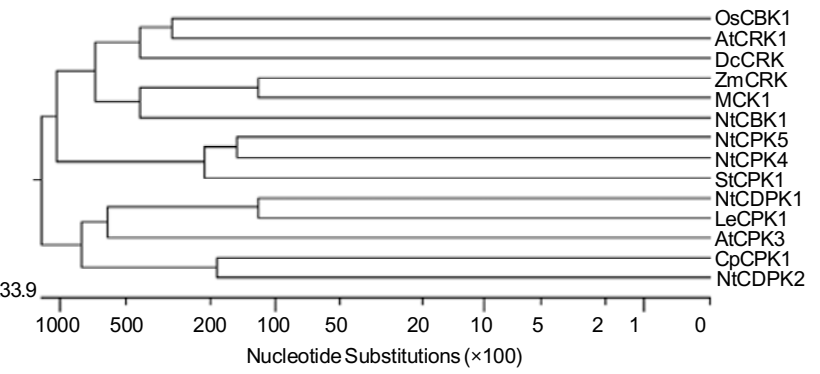

tains the N-terminal region and the kinase domain, but lacking the junction domain and the calmodulin-like domain. It has the full autophosphorylation and kinase activities (Fig. 3D). P1's activity was however much lower than NtCPK5's, but similar to NtCDPK1 (date not shown). Differing from the full-length NtCPK5, the phosphorylation activity of $\mathrm{P} 1$ does not respond to $\mathrm{Ca}^{2+}$ by showing similar activities in the presence of both $\mathrm{CaCl}_{2}$ and EGTA,

Fig. 2 Alignment of the predicted amino acid sequence of NtCPK5 with other plant protein kinases. (A) Phylogenic tree of NtCPK5 and other protein kinases. (B) Alignment of the predicted amino acid sequence of NtCPK5 with NtCPK4, StCPK1 and NtCDPK1. Identities between these kinases are indicated by shade squares. Gaps were indicated by dashes (-). The eleven subdomains in kinase domain are indicated. Asterisks (*) indicate residues that are conserved in all Ser/Thr kinases. The N-terminal presumed myristoylation site and continuous six threonines, junction domain and C-terminal four EF-hand loops are marked with a box. Genebank accession number: OsCBK1 (AF368282), AtCRK1 (AF435448), DcCRK (X83869), ZmCRK (D84507), MCK1 (1839597), NtCBK1 (AF435450), NtCPK5 (AY971376), NtCPK4 (AF435451), StCPK1 (AF030879), NtCDPK1 (AF072908), LeCPK1 (AJ308296), AtCPK3 (AL035394), CpCPK1 (U90262), NtCDPK2 (AJ344154). 
A

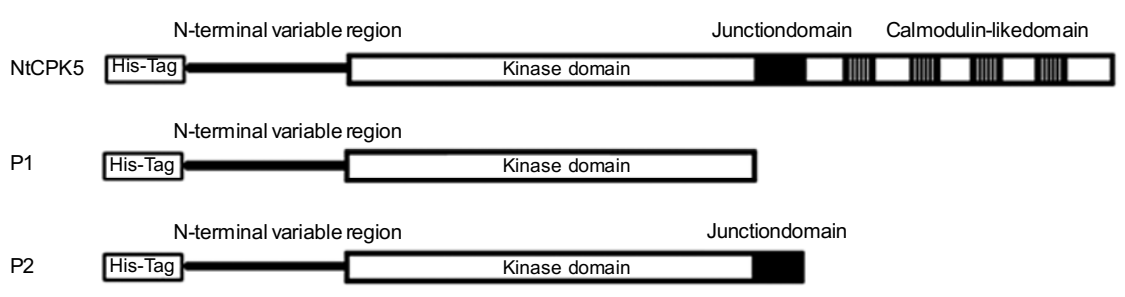

B

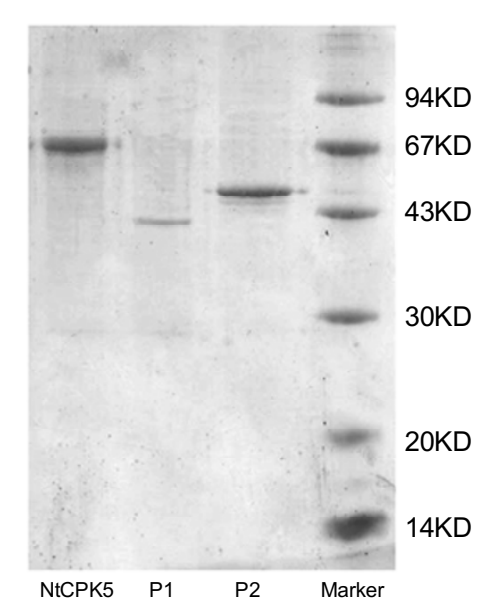

D

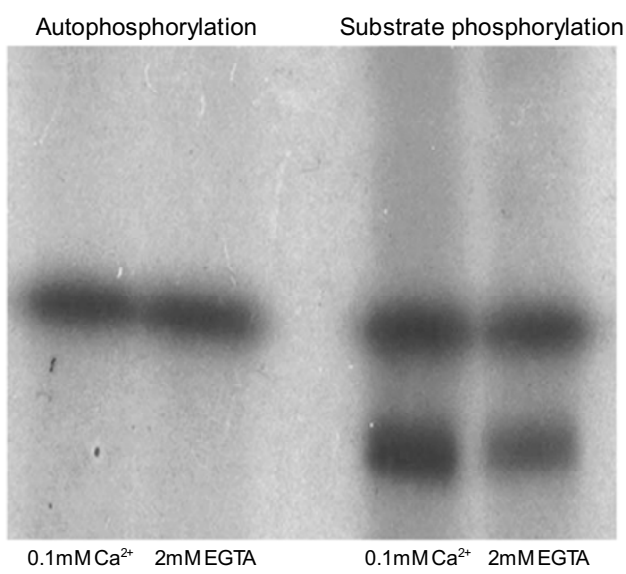

C

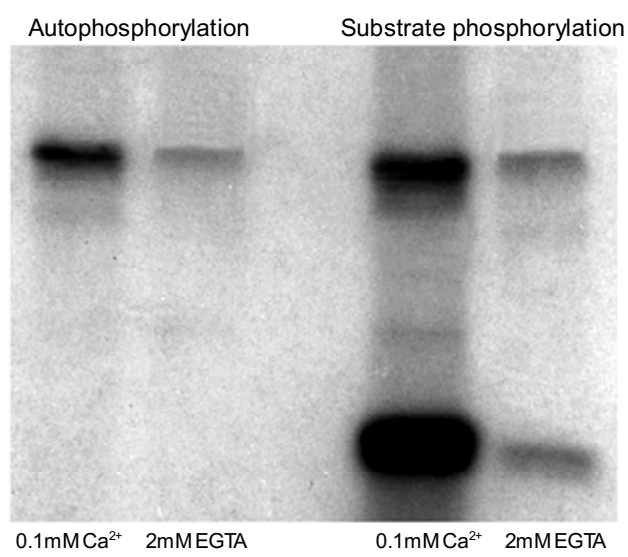

E

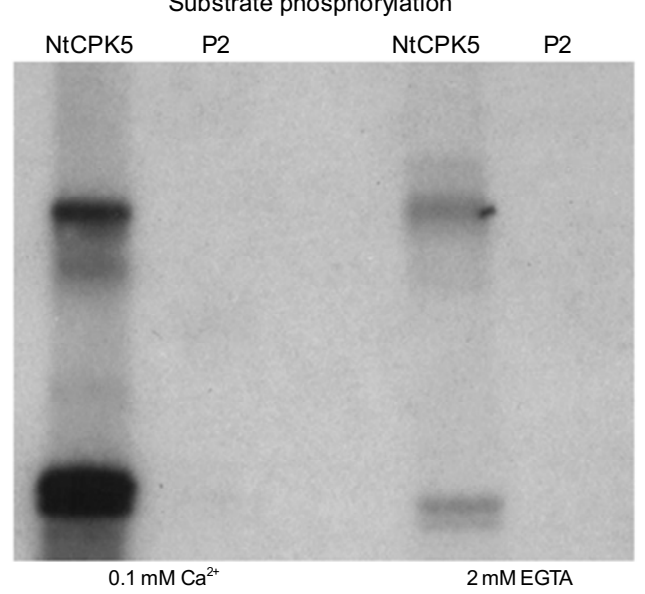

Fig. 3 Analyses of kinase activity of the recombinant NtCPK5 and two truncated proteins P1 and P2. (A) Diagrams of full-length and truncated forms of NtCPK5, P1 and P2. (B) Expression of the recombinant NtCPK5, P1 and P2 from sf-9 cells. After SDSPAGE, the purified recombinant proteins were visualized with Coomassie brilliant blue staining. Molecular size markers are indicated on the right. (C) Autophosphorylation and substrate phosphorylation (1 mg/ml histone IIIs) of recombinant NtCPK5. (D) Autophosphorylation and substrate phosphorylation $(1 \mathrm{mg} / \mathrm{ml}$ histone IIIs) of P1. (E) Substrate phosphorylation (1 mg/ml histone IIIs) of recombinant NtCPK5 and P2. $100 \mathrm{ng}$ of the purified recombinant proteins were subjected to kinase reactions in the presence of $0.1 \mathrm{mM} \mathrm{Ca}^{2+}$ or $2 \mathrm{mM}$ EGTA (for autophosphorylation), or plus $1 \mathrm{mg} / \mathrm{ml}$ histone IIIs (for substrate phosphorylation), and electrophoresed on SDS-PAGE gels. The gel was vacuum-dried and exposed to x-ray film at $-80^{\circ} \mathrm{C}$.

suggesting the regulatory role of its C-terminal calmodulinlike domain in calcium signaling pathway (Fig. 3D). Surprisingly, P2, which is $\mathrm{P} 1$ plus the junction domain does not show detectable kinase activity regardless of the presence or absence of $\mathrm{Ca}^{2+}$ (Fig. 3E), suggesting that the junction domain may be a negative regulatory domain of the kinase. This result is supporting the model of CPKs activation that the binding of $\mathrm{Ca}^{2+}$ can trigger conformational change of the kinase to release an auto-inhibitor from the active site [25]. In the absence of the calcium-binding 
domain, access to the active site is permanently blocked by the pseudosubstrate auto-inhibitor rendering the enzyme inactive [6].

\section{Characterization of the kinetic properties of NtCPK5}

To investigate the optimum conditions for NtCPK5's enzymatic activity, a range of $\mathrm{Mg}^{2+}$ concentration $(0-25 \mathrm{mM})$ and of $\mathrm{pH}(6-9.5)$ was tested. Using histone IIIs as substrates, the optimum concentration of $\mathrm{Mg}^{2+}$ for $\mathrm{NtCPK} 5$ activity was $10 \mathrm{mM}$ (Fig. 4A). The kinase activity of $\mathrm{NtCPK} 5$ was reduced to $51 \%$ of the maximum when $\mathrm{Mg}^{2+}$ concentration was used at $20 \mathrm{mM}$. Maximum activity of $\mathrm{NtCPK} 5$ was observed at $\mathrm{pH}$ 8.0. An acidic condition of $\mathrm{pH} 6.0$ and a basic condition of $\mathrm{pH} 9.5$ reduced the kinase activity to $34 \%$ and $60 \%$ respectively. The $\mathrm{pH}$-oriented activity of NtCPK 5 was similar to that observed for OsCBK in rice [12]. NtCPK5 can phosphorylate histone IIIs rapidly and reached about $60 \%$ of the maximal activity within $10 \mathrm{~min}$ in the presence of $0.1 \mathrm{mM} \mathrm{CaCl}{ }_{2}$ (Fig. 4C). In the presence of $2 \mathrm{mM}$ EGTA, the enzyme remained the minimum activity (Fig. 4C).
To analyze the effect of $\mathrm{Ca}^{2+}$ on NtCPK5's activity, a range of $\mathrm{Ca}^{2+}$ concentration was used for the activity assay (Fig. 4D). The kinase activity of NtCPK 5 on histone IIIs was stimulated by increasing amount of $\mathrm{Ca}^{2+}$ with one-half maximal activation $\left(K_{0.5}\right) 0.04 \mu \mathrm{M}$, while on syntide-2 with one-half maximal activation of $0.06 \mu \mathrm{M}$. Thus NtCPK 5 behaves similarly to other plant CPKs in terms of their $\mathrm{Ca}^{2+}$-dependent and stimulating activity [26], probably via their highly conserved $\mathrm{Ca}^{2+}$-binding domain.

The kinase activities of NtCPK 5 on histone IIIs or syntide- 2 were further analyzed by using different concentrations of the substrates and fitted to Lineweaver-Burk curve (Fig. 5). For histone IIIs, $K_{m}$ value of $210 \mu \mathrm{g} / \mathrm{ml}$ and $V_{\max }$ of $526 \mathrm{nmol} / \mathrm{min} / \mathrm{mg}$ were observed (Fig. 5A). For syntide-2, a $K_{m}$ value of $30 \mu \mathrm{M}$ and $V_{\max }$ value of 2008 $\mathrm{nmol} / \mathrm{min} / \mathrm{mg}$ were observed (Fig. 5B). A fixed ATP concentration $(50 \mu \mathrm{M})$ was used in the reaction.

\section{Subcellular localization of NtCPK5}

The N-terminal variable region has been shown essential for the subcellular localization of many CPKs [7-10].
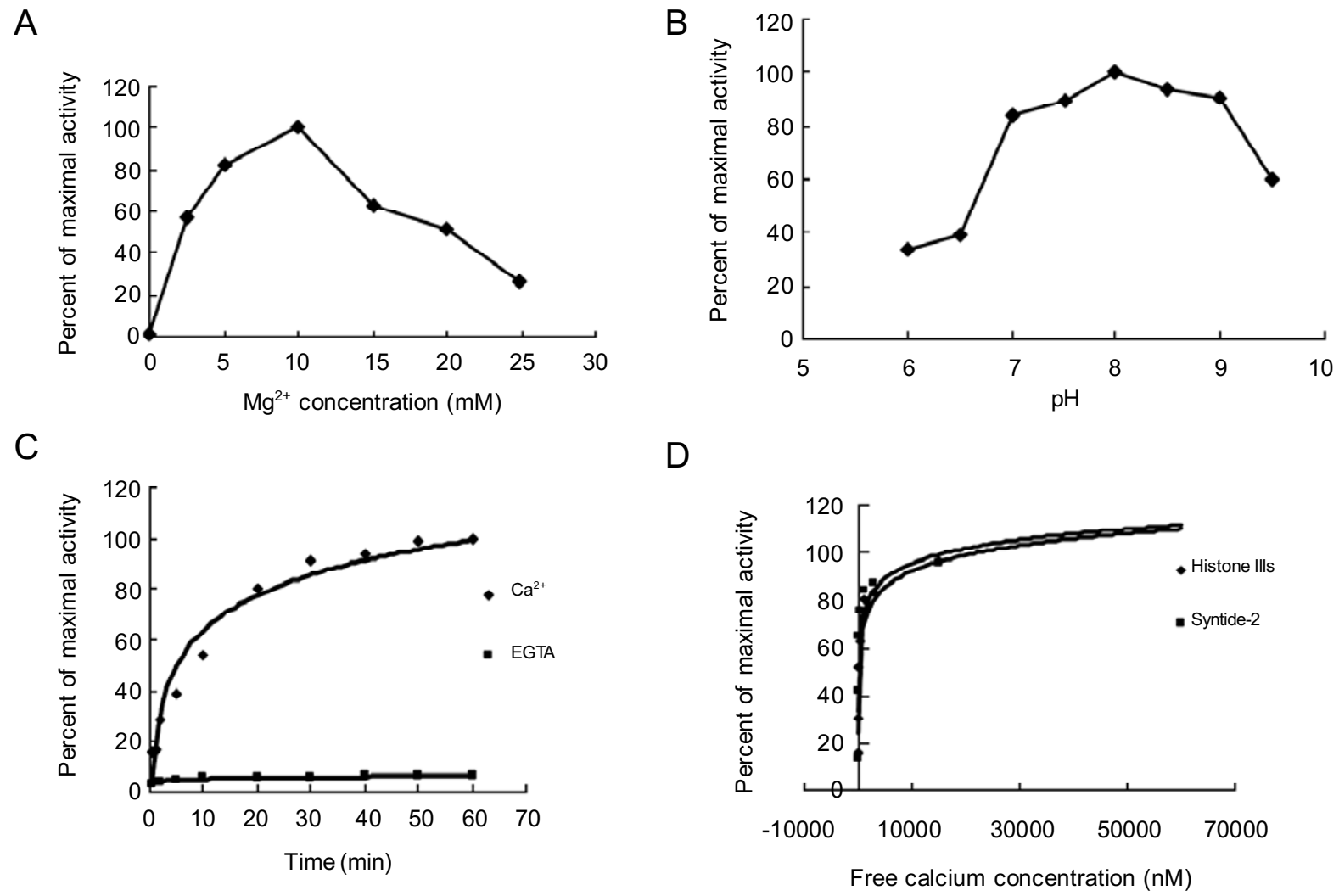

Fig. 4 Analysis of the kinetic properties of NtCPK5. (A) Effects of $\mathrm{Mg}^{2+}$ and (B) pH concentration on NtCPK5 activity. (C) Time

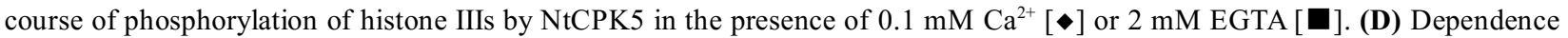
on $\mathrm{Ca}^{2+}$ concentration for protein kinase activity of NtCPK5. The enzyme activity was assayed with a histone IIIs [ $]$ or syntide2 [ ] ] as substrate in the presence of various concentrations of calcium ions. 

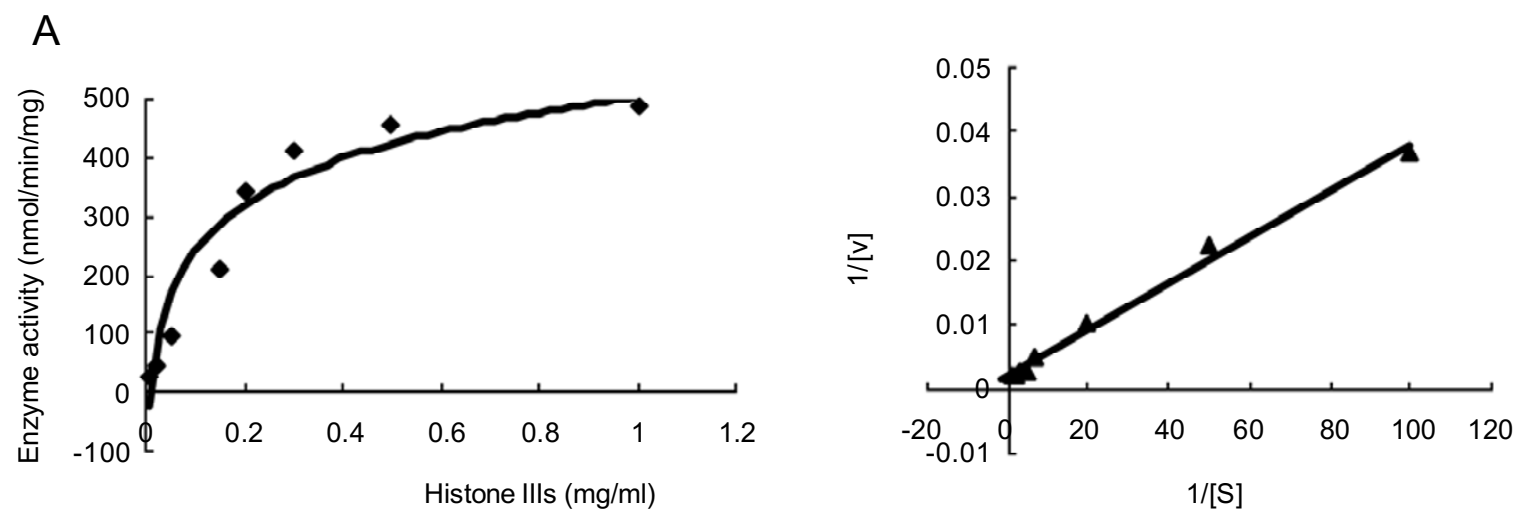

B
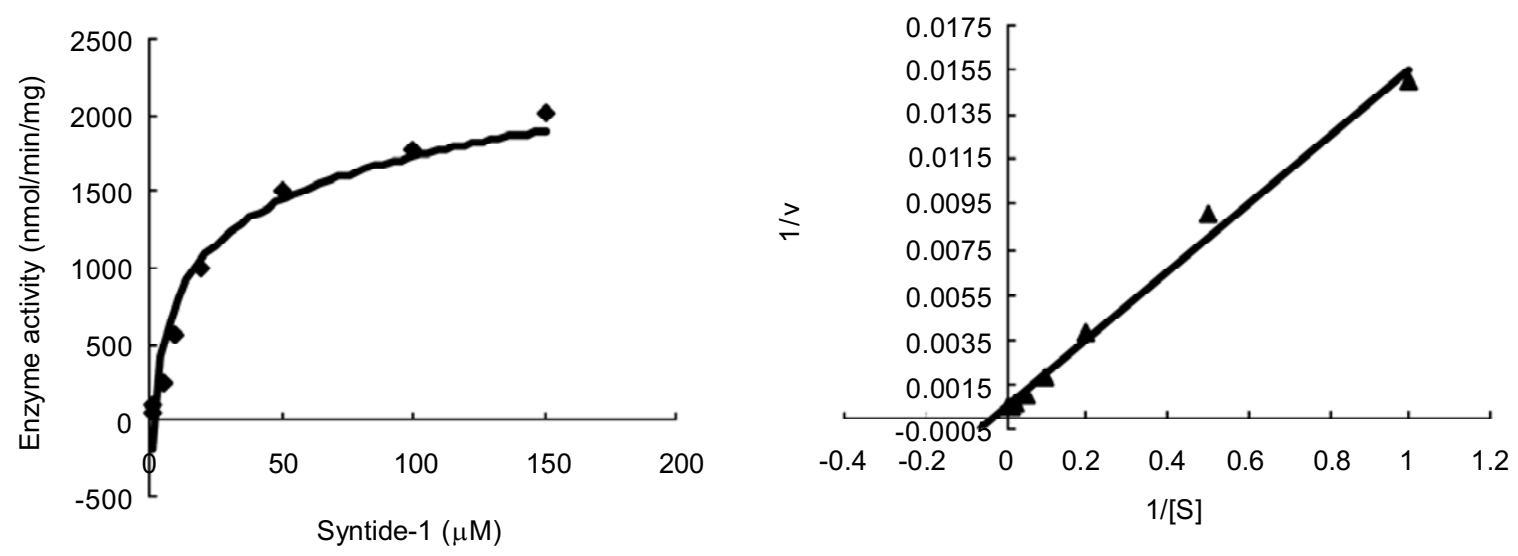

Fig. 5 Determination of $K_{m}$ and $V_{\max }$ values for NtCPK5 using different concentration of substrate. The protein kinase activity was assayed in the presence of various concentrations of histone IIIs (A) and syntide-2 (B) as substrate. The right shows a LineweaverBurk plot for the same values.

The N-terminal acylation is critical for membrane-anchor of CPKs. NtCPK4 and NtCPK5 are high homologous in tobacco but their $\mathrm{N}$-termini are obviously diverse. NtCPK 5 but not NtCPK4 has putative $\mathrm{N}$-terminal myristoylation site by ScanProsite searching of PlantsP database. The Cys at the $4^{\text {th }}$ position from $\mathrm{N}$-terminus as a presumed palmitoylation site in NtCPK 5 is replaced by Ala. It is likely that these two kinases have different cellular localizations. In order to analyze the role of CPKs' N-termini in subcellular distribution of these two CPKs, GFP fusion proteins of NtCPK4/GFP and NtCPK5/GFP and three mutant proteins of NtCPK5/GFP with mutated acylation sites were generated and transiently expressed in onion epidermal cells by bombardment.

Confocal microscopy showed that NtCPK5/GFP was efficiently targeted to the cell periphery, suggesting the plasma membrane localization of NtCPK5/GFP (Fig. 6A). $\mathrm{NtCPK} 4 / \mathrm{GFP}$ was distributed throughout the cell including the cytoplasm and the nucleus (Fig. 6B), indistinguish- able from that of GFP (Fig. 6F). Because NtCPK5 had putative $\mathrm{N}$-terminal acylation sites which are missing in $\mathrm{NtCPK} 4$, we assume that these sites played vital roles in differential subcellular localizations between NtCPK5 and $\mathrm{NtCPK} 4$. To test this hypothesis, three mutant proteins were made by site-directed mutagenesis as described in Material and Methods. To summarize, mutations of Gly2Ala or Cys4Ala or a double mutant of Gly2Ala/ Cys4Ala were made, fused to GFP and transiently expressed in onion epidermal cells (Fig. 6C-E). Analysis by confocal microscopy showed that all these mutation forms lose their plasma membrane localization, instead a distribution throughout the cells was observed, suggesting the importance of both $\mathrm{N}$-terminal myristoylation and palmitoylation sites for plasma membrane targeting of NtCPK 5 in onion cells. Nmyristoylation or palmitoylation alone is not sufficient for NtCPK 5 membrane localization and both amino acids Gly2 and Cys 4 are required for the plasma membrane localization of NtCPK5. 

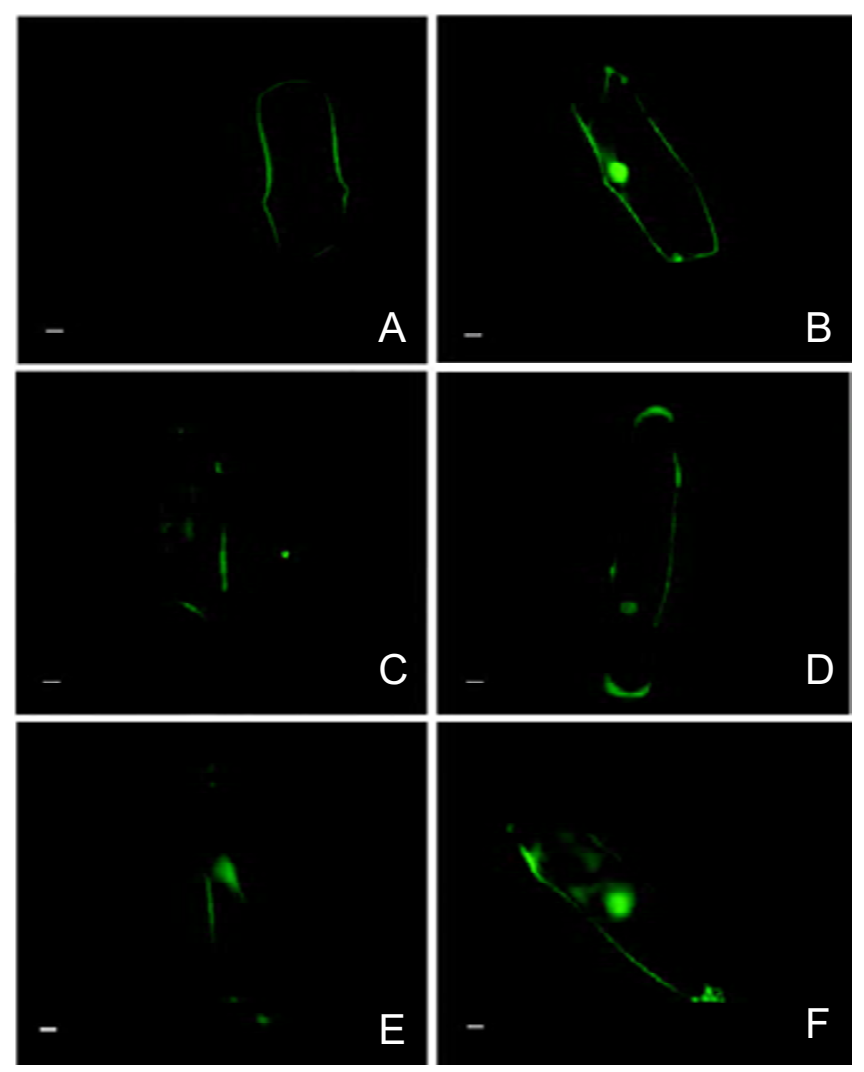

G

CPK

$\begin{array}{ll}\text { Wild } & \text { NtCPK4 MGNNCF S - } \\ \text { Wild } & \text { NtCPK5 MG S C F S S S- } \\ \text { Myr- } & \text { NtCPK5 MA S C F S S S- } \\ \text { Pal- } & \text { NtCPK5 MG SAF S S S- } \\ \text { Myr/Pal- NtCPK5 MA } & *{ }^{*} \text { A F S S S- }\end{array}$

Fig. 6 Subcellular localization of NtCPK5/GFP and NtCPK4/GFP fusion proteins. Wild-type NtCPK5 and N-terminal myristoylation and palmitoylation mutants were transiently expressed as C-terminal GFP-fusion proteins in onion epidermal cells. (A) Localization of wild NtCPK5/GFP fusion protein. (B) Localization of wild $\mathrm{NtCPK} 4 / \mathrm{GFP}$ fusion protein. (C) Localization of mutated myristoylation site Gly2Ala NtCPK5/GFP fusion protein. (D) Localization of mutated palmitoylation site Cys4Ala NtCPK5/GFP fusion protein. (E) Localization of mutated myristoylation and palmitoylation sites Gly2Ala/Cys4Ala NtCPK5/GFP fusion protein. (F) Control. Soluble localization of GFP protein. (G) Diagrams of wild NtCPK4/GFP, NtCPK5/GFP and mutant NtCPK5/ GFP. The length of the bars corresponds to $10 \mu \mathrm{m}$.

\section{DISCUSSION}

In this study, a tobacco cDNA designated as NtCPK 5 was cloned and characterized. Analysis of amino acid sequence indicates that it could be a calcium-dependent protein kinase, since it contains all the features of $\mathrm{Ca}^{2+}$-dependent protein kinases, including conserved kinase domain, junction domain and CaM-like domain. Searching the PlantsP database with NtCPK 5 amino acid sequence [19], it was found of a protein kinase catalytic domain (pfscan), four EF-hand binding $\mathrm{Ca}^{2+}$ motives (ScanProsite), a protein kinase ATP-binding region signature (ScanProsite), a Ser/Thr protein kinase active-site signature (ScanProsite) and seven potential N-myristoylation sites (ScanProsite). Compared to other CPKs, NtCPK5 showed high homology to that of NtCPK4 and potato StCPK1 [20], especially to that of NtCPK4. They differ in N-terminal variable regions of the N-termini acylation sites and the six continuous threonines from $34^{\text {th }}$ to $39^{\text {th }}$ in NtCPK5. The N-termini acylation sites have been shown required for membraneanchoring of many CPKs [7-10], while the role of the six threonines stretch in NtCPK5's N-terminal variable region is still unknown.

CPK-related protein kinases (CRKs) have both kinase domain and junction domain closely related to those of CDPKs, while their C-terminal domains have degenerated EF-hands [6]. Biochemical analyses showed that the activation of CRKs does not require calcium [13, 24, 27]. Phylogenetic analyses indicated that there was a common evolutionary origin for plant CPKs and CRKs [6]. An evolutionary link between $C P K s$ and $C R K s$, named $S t C P K 1$, has been cloned in potato plants [20]. StCPK1 shares more similarity to CRKs than to CPKs, although sequence analyses indicate that it contains conserved EF-hands at the Cterminus. NtCPK5 showed high homology to StCPK1 (89. $4 \%$, identity), NtCPK 5 could thus be considered as an evolutionary link between CPKs and CRKs similar to StCPK1.

To characterize the biochemical activity of NtCPK5 as a calcium-dependent protein kinase, both full-length and truncated NtCPK 5 were used for kinase assays. Substrate phosphorylation by full-length NtCPK 5 can be greatly stimulated by $\mathrm{Ca}^{2+}$. The $K_{0.5}$ of calcium-stimulating NtCPK5's activity is different for different substrates such as histone IIIs and syntide- 2 used. All these dada suggest that NtCPK5 is a new member of the CPK subfamily in tobacco.

Previous reports have shown that many CPKs were modified by $\mathrm{N}$-acylation including myristoylation and palmitoylation and targeted to membrane [7-10]. The Nmyristoylation site was a conserved $\mathrm{N}$-terminal sequence of 7-10 aa long signature as $\mathrm{MGxxxS} / \mathrm{T}(\mathrm{K})$ [28]. In our experiments, NtCPK5/GFP was targeted to the plasma membrane, while NtCPK4/GFP was distributed within the whole cell. This could due to the putative N-terminal acy- 
lation sites in NtCPK5. When the N-acylation sites (Gly2Ala and Cys4Ala) were mutated, NtCPK5/GFP lost plasma membrane localization.

In summary, a new CPK isoform NtCPK 5 from tobacco was cloned and characterized. NtCPK 5 behaviors like a typical calcium-dependent protein kinase. NtCPK5 localized to the plasma membrane in onion cells, while $\mathrm{NtCPK} 4$, a homologue of NtCPK 5 was distributed evenly within the cell, suggesting that the $\mathrm{N}$-terminal sequence of the two NtCPKs plays vital roles in their subcellular localization. An understanding of the role of acylation in $\mathrm{NtCPK} 5$ activity needs further analysis of the physiological function of NtCPK5 and its mutant proteins in plants.

\section{ACKNOWLEDGEMENTS}

This work was supported in part by the National Natural Science Foundation of China (Grant No. 30230050) and the Program for Changjiang Scholars and Innovative Research Team in University to Ying Tang LU.

Received, May 10, 2005

Revised, July 26, 2005

Accepted, Aug 15, 2005

\section{REFERENCES}

1 Reddy ASN. Calcium: silver bullet in signaling. Plant Sci 2001; 160:381-404.

2 Cheng SH, Willmann MR, Chen H, Sheen J. Calcium Signaling through Protein Kinases. The Arabidopsis Calcium-dependent Protein Kinase Gene Family. Plant Physiol 2003; 129:469-85.

3 Zhang L, Lu YT. Calmodulin-binding protein kinase in plants. Trends Plant Sci 2003; 8:123-7.

4 Rudd JJ, Franklin-Tong VE. Unravelling responsespecificity in $\mathrm{Ca}^{2+}$ signaling pathways in plant cells. New Phytol 2001; 151:733 .

5 Hrabak EM. Calcium-dependent protein kinases and their relatives. Advances in botanical sciences, plant protein kinases. New York: Academic Press 2000; 32:185-223.

6 Harmon AC, Gribskov M, Harper JF. CDPKs: a kinase for every $\mathrm{Ca}^{2+}$ signal? Trends Plant Sci 2000; 5:154-9.

7 Martin ML, Busconi L. Membrane localization of a rice calciumdependent protein kinase (CDPK) is mediated by myristoylation and palmitoylation. Plant J 2000; 24:429-35.

8 Rutschmann F, Stalder U, Piotrowski M, Oecking C, Schaller A. LeCPK1, a calcium-dependent protein kinase from tomato plasma membrane targeting and biochemical characterization. Plant Physiol 2002; 129:156-68.

9 Raíces M, Gargantini PR, Chinchilla D, et al. Regulation of CDPK isoforms during tuber development. Plant Mol Biol 2003; 52: 1011-24.

10 Dammann C, Ichida A, Hong B, et al. Subcellular targeting of nine Calcium-dependent protein kinase isoforms from Arabidopsis. Plant Physiol 2003; 132:1840-8.
11 Lu YT, Hidaka H, Feldman LJ. Characterization of a calcium/ calmodulin-dependent protein kinase homolog from maize roots showing light- regulated gravitropism. Planta 1996; 199:18-24.

12 Zhang L, Liu BF, Liang S, Jones RL, Lu YT. Molecular and biochemical characterization of a calcium/calmodulin-binding protein kinase from rice. Biochem J 2002; 368:145-57.

13 Wang Y, Liang S, Xie QG, Lu YT. Characterization of a calmodulin-regulated $\mathrm{Ca}^{2+}$-dependent-protein-kinase-related protein kinase AtCRK1 from Arabidopsis. Biochem J 2004; 383: 73-81.

14 Hua W, Liang S, Lu YT. A tobacco (Nicotiana tabaccum) calmodulin-binding protein kinase, $\mathrm{NtCBK} 2$, is regulated differentially by calmodulin isoforms. Biochem J 2003; 376:291-302.

15 Ma L, Liang S, Jones RL, Lu YT. Characterization of a novel calcium/calmodulin-dependent protein kinase from tobacco. Plant Physiol 2004; 135: 1280-93.

16 Bers DM, Patton CW, Nuccitelli R. A Practical Guide to Preparation of $\mathrm{Ca}^{2+}$ Buffers. Methods Cell Biol 1994; 40:3-29.

17 Hua W, Zhang L, Liang S, Jones RL, Lu YT. A Tobacco Calcium/ Calmodulin-binding Protein Kinase Functions as a Negative Regulator of Flowering. J Biol Chem 2004; 279:31483-94.

18 Scott A, Wyatt S, Tsou PL, Robertson D, Allen NS. Model system for plant cell biology: GFP imaging in living onion epidermal cells. Biotechniques 1999; 26:1125-32.

19 Gribskov M, Fana F, Harper J, et al. PlantsP: a functional genomics database for plant phosphorylation. Nucleic Acids Res 2001; 29:111-3.

20 Lakatos L, Hutvagner G, Banfalvi Z. Potato protein kinase StCPK1: a putative evolutionary link between CDPKs and CRKs. Biochim Biophys Acta 1998; 1442:101-8.

21 Yoon GM, Cho HS, Ha HJ, Liu JR, Lee Pai HS. Characterization of NtCDPK1, a calcium-dependent protein kinase gene in Nicotiana tabacum, and the activity of its encoded protein. Plant Mol Biol 1999; 39:991-1001.

22 Romeis T, Ludwig AA, Martin R, Jones JDG. Calcium- dependent protein kinases play an essential role in a plant defence response. EMBO J 2001; 20:5556-67.

23 Mary EI, Hopkins RB, White TJ, Lomax TL. Cloning, expression and $\mathrm{N}$-terminal myristoylation of $\mathrm{CpCPK} 1$, a calcium-dependent protein kinase from zucchini (Cucurbita pepo L.). Plant Mol Biol 1999; 39:199-208.

24 Furumoto T, Ogawa N, Hata S, Izui K. Plant calcium-dependent protein kinase-related kinases (CRKs) do not require calcium for their activities. FEBS Lett 1996; 396:147-51.

25 Harper JF, Sussman MR, Schaller GE, et al. A calciumdependent protein kinase with a regulatory domain similar to calmodulin. Science 1991; 252:951-4.

26 Chehab EW, Patharka OR, Hegeman AD, Taybi T, Cushman JC. Autophosphorylation and subcellular localization dynamics of a salt- and water deficit-induced Calcium-dependent protein kinase from ice plant. Plant Physiol 2004; 135:1430-46.

$27 \mathrm{Du} \mathrm{W}$, Wang Y, Liang S, Lu YT. Biochemical and expression analysis of an Arabidopsis Calcium-dependent protein kinaserelated kinase. Plant Sci 2005; 168:1181-92.

28 Thompsona Jr GA, Okuyamab H. Lipid-linked proteins of plants. Prog Lipid Res 2000; 39:19-39. 